\title{
FAMILIAL PAGET'S DISEASE WITH EARLY ONSET
}

\author{
R. E. IRvine, London, England
}

In 1889 Paget wrote of osteitis deformans: "I have tried in vain to trace any inherited tendency to the disease. I have not found it twice in the same family." Nevertheless it is now accepted that Paget's disease may run in families, though uncommonly. The family with Paget's disease reported here consists of a father and two daughters. The father's case is of interest because of the rare type of malignant change which led to his death, and the affection in the two daughters is notable for the unusually early onset at eighteen years. There are three other siblings, two brothers and a sister, who are all in the fourth decade of life. None presents any clinical abnormality and radiographic examination of their skulls and pelves showed no skeletal changes.

\section{CASE REPORTS}

Case 1-The father of the family suffered from Paget's disease from about the age of thirty-five. The disease was noticed first in the right tibia, but later affected almost the entire skeleton, including the skull and jaws. The patient died at the age of sixty-five from malignant changes in the mandible. A detailed account of this case with illustrative radiographs is given elsewhere in this issue by Cones.* Case 2-The eldest daughter of the patient described in Case 1 is now aged forty. At the age of eighteen years she noticed a painless swelling in the roof of the mouth, for which she consulted a dental surgeon. The swelling has not changed in the twenty-two years since its onset. Recent examination revealed a bony swelling of the left side of the hard palate and of the alveolar process of the maxilla, more marked on the left but extending across the mid-line (Fig. 1). The rest of the skeleton was clinically normal. There was no pigmentation of the skin.

Radiographic examination confirmed the bony thickening of the palatal and alveolar processes of the maxilla. There was alteration of bone structure, the changes being mainly sclerotic and hyperostotic (Fig. 2). In addition there was a large area of osteoporosis circumscripta, mainly in the left frontal region of the skull (Fig. 3). Serial radiographs have shown an extension of this lesion during the last two years but there are not as yet any sclerotic changes in it. The rest of the skeleton has remained radiographically normal except for the pelvis, which shows thickening and increased density above the right acetabulum, with coarsening of the trabecular pattern in the right ischio-pubic ramus, typical of Paget's disease (Fig. 4). Biochemical investigations showed a slightly raised serum alkaline phosphatase of $17 \cdot 2 \mathrm{King}$ Armstrong units. The serum calcium and phosphorus were normal. The patient has three sons aged twelve, ten and seven years who present no abnormalities of the skeleton.

Case 3-The youngest daughter of the family is aged thirty-two. When she was eighteen she first noticed a painless swelling of the right lower jaw. This gradually increased in size until by the age of twenty-six years the whole lower jaw was thickened and the left upper jaw was beginning to enlarge as well. Examination at that time (1945) revealed a thickening of the whole mandible, especially on the right, and of the alveolar process of the left maxilla (Fig. 5). Distended veins were visible in the skin beneath the chin. There was no pigmentation of the skin. Radiographs showed that the whole mandible was affected by changes of a hyperostotic and porotic type with occasional patches of sclerosis (Fig. 7). Similar changes were present in the left maxilla (Fig. 8). In the right cranial vault there was an area of osteoporosis about an inch in diameter. The rest of the skeleton was normal. Biochemical investigations-The serum calcium and phosphorus were within normal limits but the alkaline phosphatase was raised to 45 King Armstrong units.

Treatment-In 1946, after preliminary radiotherapy, a cosmetic operation was performed by Professor Rushton and Sir Harold Gillies, parts of the maxilla and mandible being sliced off to restore a normal contour. Marked improvement was secured (Fig. 6).

Histological examination-The operation enabled fragments of bone to be examined microscopically. Sections from the maxilla (Fig. 11) and mandible (Fig. 12) showed absorption of cortical bone by numerous osteoclasts and its replacement by fine connective tissue composed

* D. M. T. Cones: An Unusual Bone Tumour Complicating Paget's Disease, page 101. 


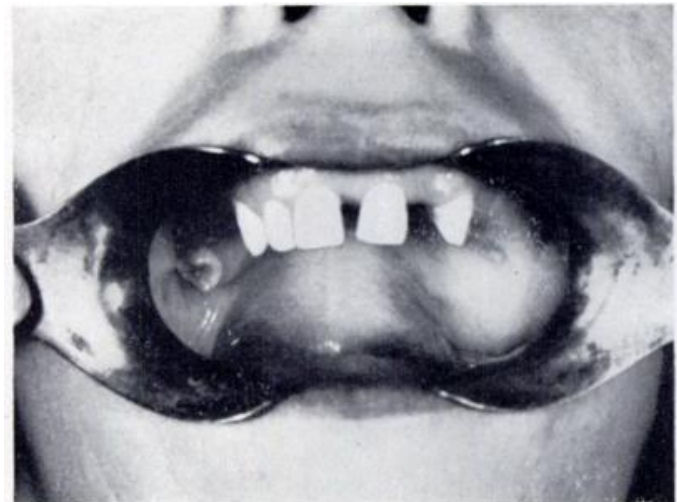

FIG. 1

Case 2-Showing the swelling of the hard palate on the left and the thickening of the alveolar process of the maxilla.

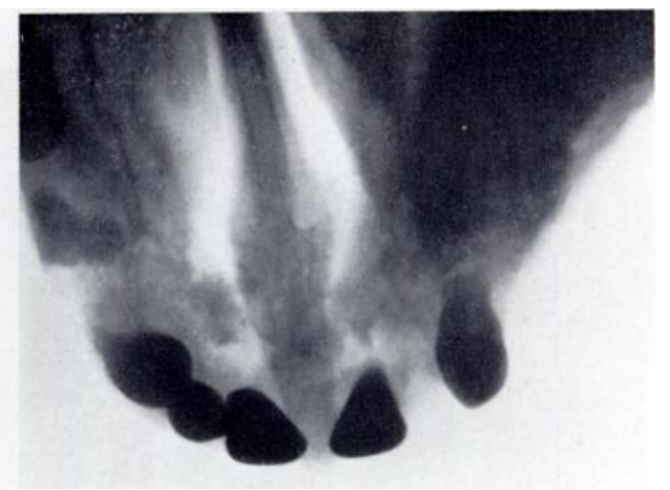

FIG. 2

Case 2-Radiograph of palate and alveolar process showing altercd bone structure with hyperostosis and sclerosis.

FIG. 3

Case 2-Lateral view of skull showing osteoporosis circumscripta extending backwards from the frontal region.

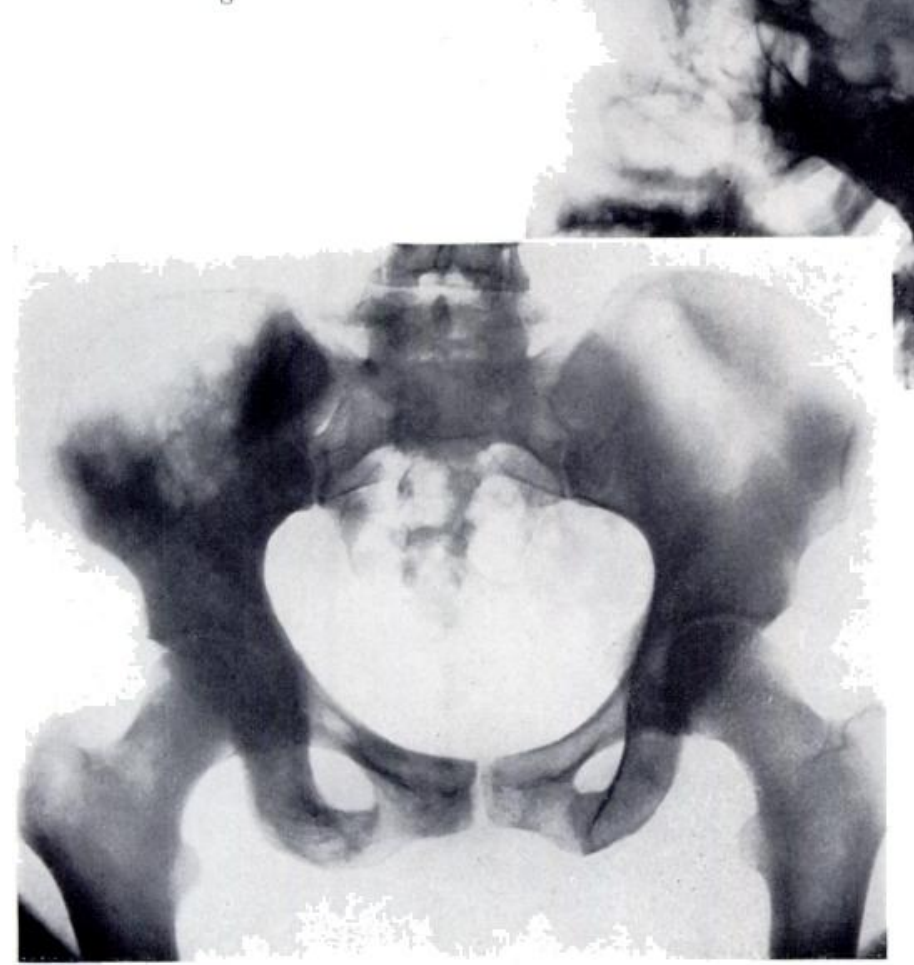

Fig. 4

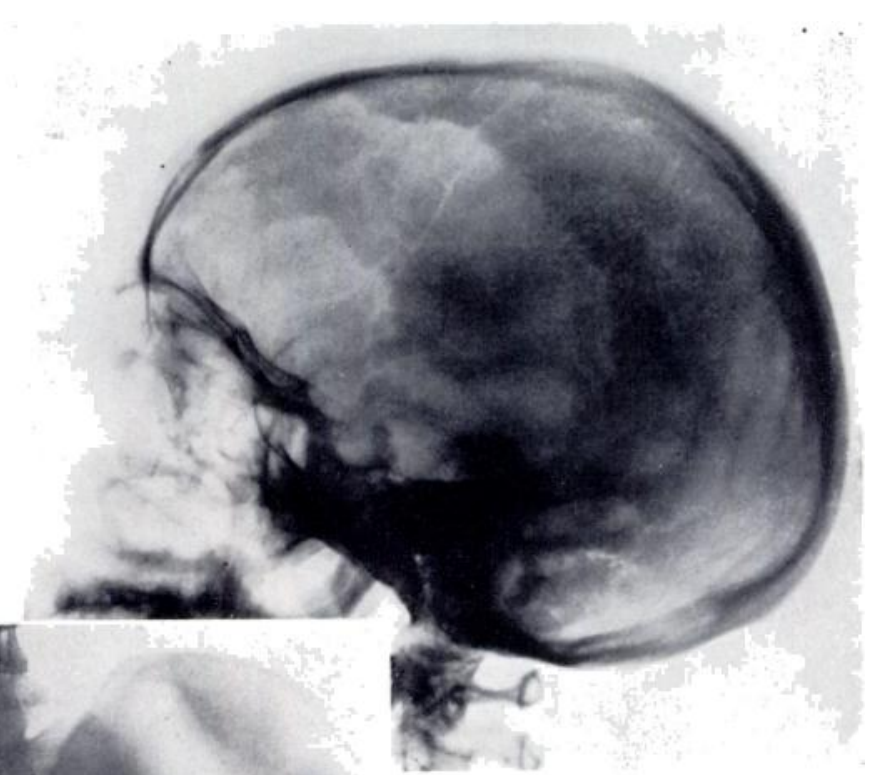

FIG. 3

FIG. 4

Case 2-Sclerosis of right ilium and thickening of right ischiopubic ramus with coarsening of the trabecular pattern. Note small area of osteoporosis in the right superior pubic ramus.

VOL. 35 B, No. 1, FEBRUARY 1953 
of spindle cells and stellate cells. Embedded in this fibrous tissue were innumerable small bone nodules of inactive appearance (Fig. 13). Figure 11 shows the formation of new osteoid trabeculae. Evidence of past bone absorption and replacement was seen in the mosaic markings shown in Figure 12. The histological picture was one of destruction of bone and its replacement by fibrous marrow with some evidence of repair and new bone formation. These appearances would do equally well for fibrous dysplasia or for Paget's disease.

Progress-Observation over six years has revealed little change in the jaws. On two occasions a regular evening temperature of 99 degrees Fahrenheit has been noted. The radiographic changes in the jaws have not altered, but the area of osteoporosis circumscripta in the skull has extended to involve large parts of the right parietal, occipital and frontal bones, and a new separate area of osteoporosis circumscripta has appeared in left frontal bone (Figs. 9 and 10). The rest of the skeleton has remained radiographically normal. The alkaline phosphatase has remained high (45-50 units). A calcium balance test carried out in 1950 showed a slight negative balance. No other biochemical changes have been observed.

This patient has two sons aged eight and five. Both are healthy and radiographic examination of the entire skeleton revealed no abnormality in either of them.

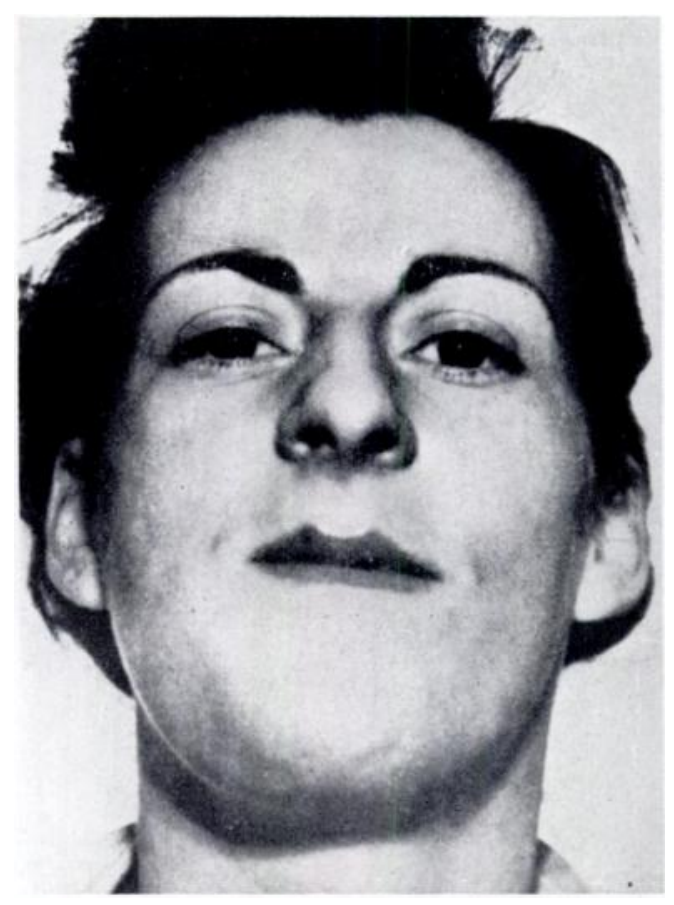

FIG. 5

Case 3-Aged twenty-six. Enlargement of the right lower jaw and some fullness of the face on the left due to enlargement of the maxilla.

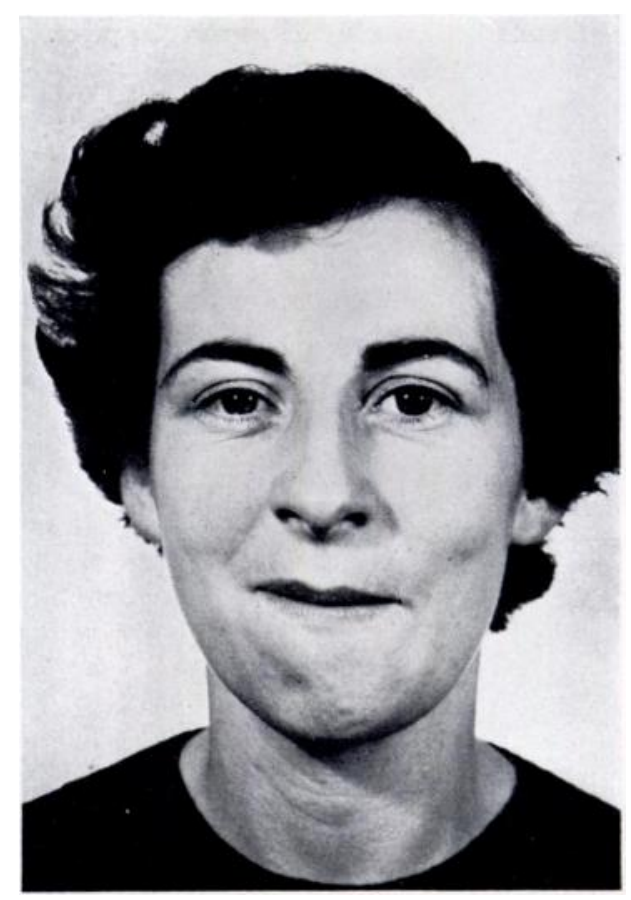

FIG. 6

Case 3-Aged thirty-one. Five years after operation, showing the improved facial appearance.

\section{DISCUSSION}

The diagnosis in the case of the father was not in doubt, but in the two daughters it was not possible at first to make a firm distinction between fibrous dysplasia and Paget's disease. The younger sister, described in Case 3, came under observation before the other members of the family, and before the osteoporosis in the cranial vault had become large enough to suggest Paget's disease. The age of onset at eighteen, the involvement of the jaws, and the radiographic appearances in the mandible all pointed to a diagnosis of fibrous dysplasia. The histological picture showed nothing that would conflict with this diagnosis, and in fact the case was reported by Rushton (1947) as one of regional osteitis fibrosa, a term which is now regarded as synonymous with the milder forms of fibrous dysplasia of bone. When it became clear that the patient was developing classical osteoporosis circumscripta the 
pessibility of Paget's disease became more likely, despite the youth of the patient. But it was not until changes strongly suggestive of Paget's disease were found in the pelvis in Case 2 that the diagnosis became reasonably certain. These changes were not observed until twenty-one years after the original manifestations in the maxilla. The similarity

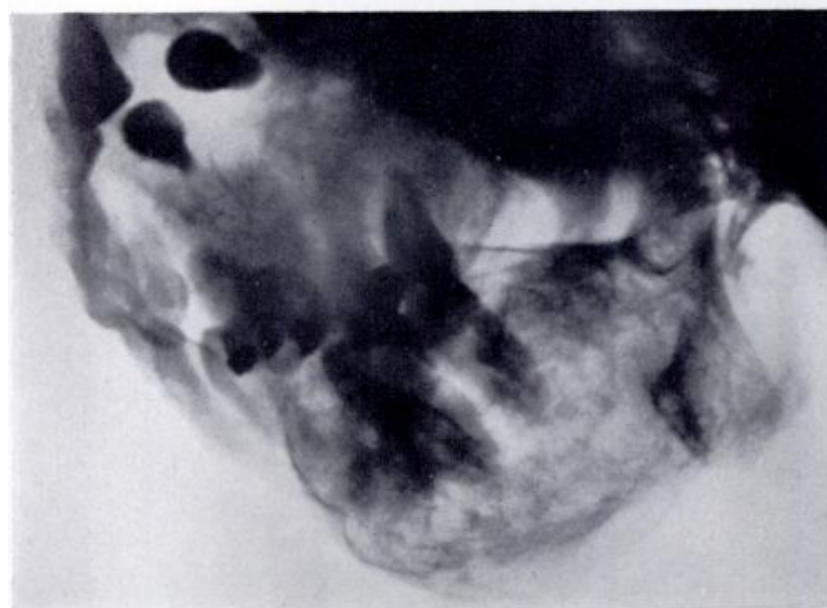

FIG. 7

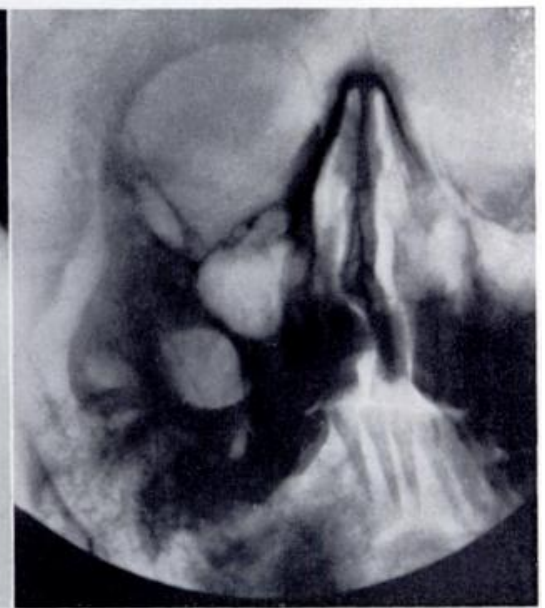

FIG. 8

Case 3. Figure 7-Nandible showing marked widening with porotic and hyperostotic changes. Figure 8Showing thickening of the left maxilla.

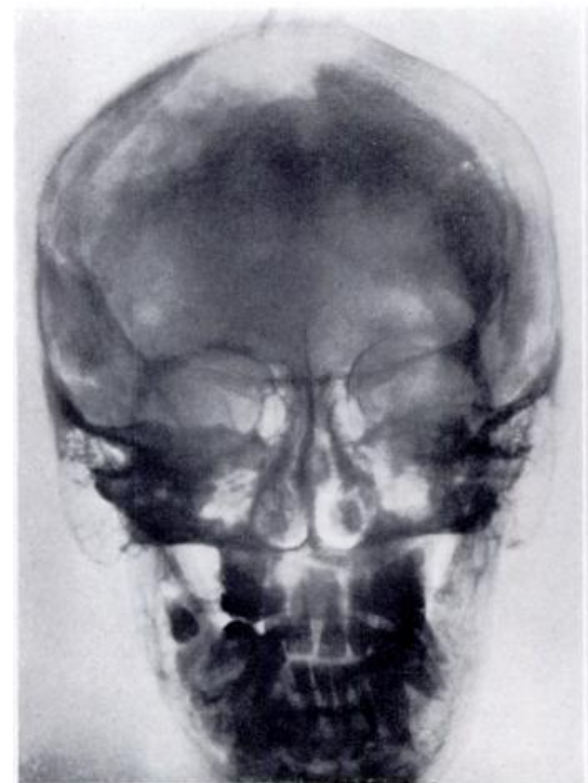

FIG. 9

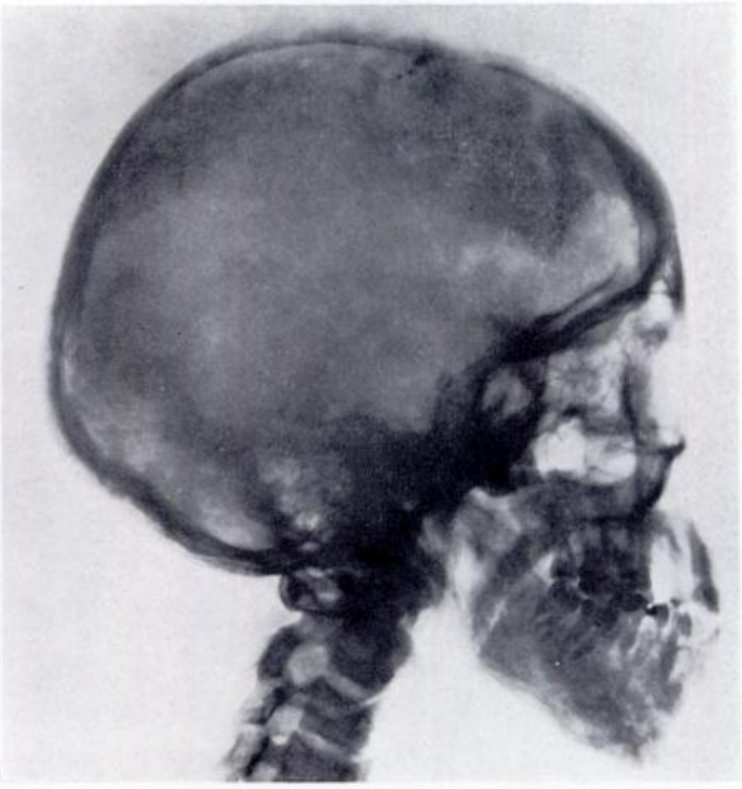

FIG. 10

Postero-anterior and lateral radiographs of skull to show the extensive osteoporosis circumscripta.

between the skeletal changes in the two sisters is so close as to make it highly probable that they both suffer from the same condition, and it now seems justifiable to regard the lesions as examples of familial Paget's disease.

Since the papers of Sosman (1927) and of Kasabach and Gutman (1937), the close VOL. $35 \mathrm{~B}$, NO. 1, FÉBRUARY 1953 
association of osteoporosis circumscripta with Paget's disease has been generally recognised. Indeed many authorities, such as Hunter and Jupe (1950), regard osteoporosis circumscripta as a specific manifestation of Paget's disease. Others, including Snapper (1949), regard it as a localised circulatory disturbance similar to the haemorrhagic infarction of the skull described

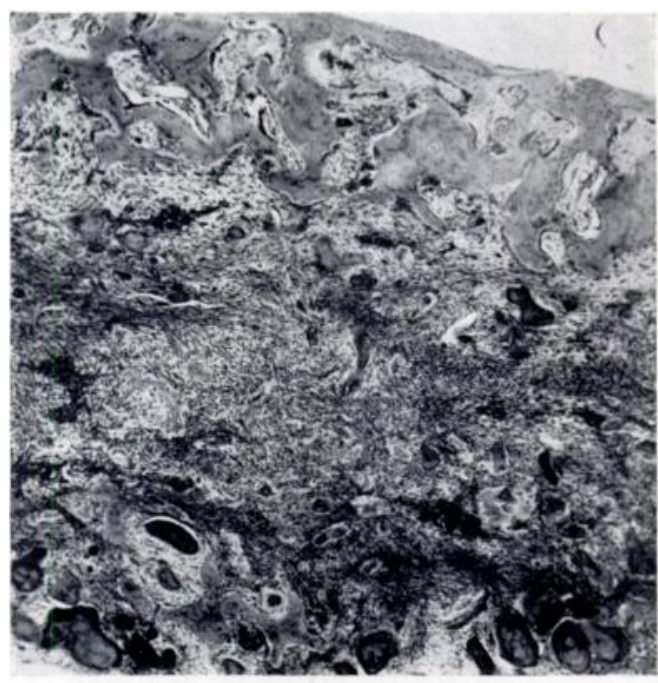

Fig. 11

Section of maxilla showing fibrous marrow rontaining bone nodules, and new osteoid trabeculae. The cortex (top) is being absorbed by osteoclasts (H. and E. 33).

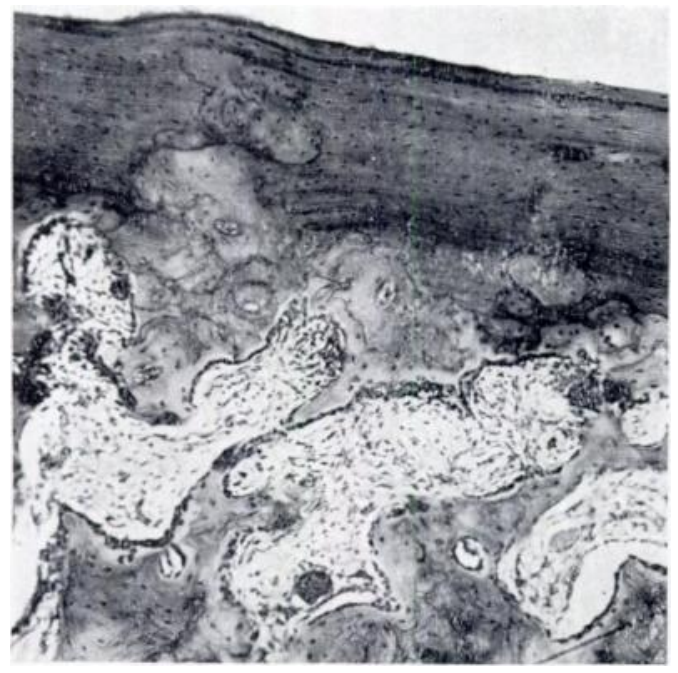

FIG. 12

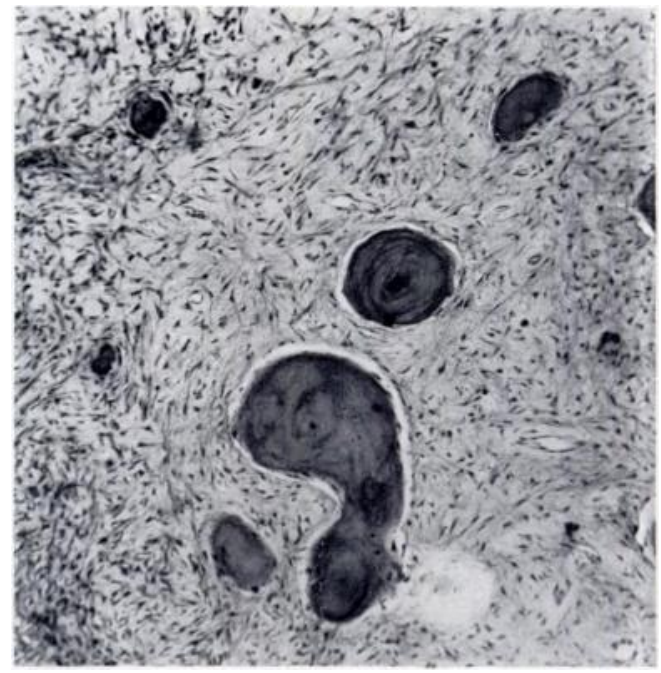

Fici. 13

Figure 12-Section of mandible. The cortex is seen with fibrous marrow below it. There are numerous giant-cell osteoclasts. Mosaic markings reveal evidence of past bone absorption and replacement (H. and E. $\times 63)$. Figure 13 -Mandible. Spindle and stellate-celled area containing inactive bone nodules (H. and E. 63).

by Schmorl (1932), and stress that although it is commonest in Paget's disease it is also found in other bone dysplasias, particularly those causing proliferation of the maxilla, and also in hyperparathyroidism. This view seems to rest largely on a paper by Windholz (1945), who described three cases of osteoporosis circumscripta; in two it was associated with 
enlargement of the maxilla, and he considered Paget's disease to be excluded in the one on histological, and in the other on radiological, grounds. The radiographic and histological changes of Paget's disease of the jaw, however, are not always pathognomonic and it is difficult to be certain that this diagnosis is really excluded in these two cases. Windholz's third case was one of hyperparathyroidism which, however, had many anomalous features and was not helped by parathyroidectomy. Snapper (1949) mentioned three cases of hyperparathyroidism with osteoporosis circumscripta but gave no details. Albright and Reifenstein (1948) stated that Paget's disease and hyperparathyroidism may occasionally occur together in the same patient. It thus seems doubtful whether osteoporosis circumscripta is due to any cause other than Paget's disease. Sosman (1927) was probably right when he considered that osteoporosis circumscripta represented the absorptive or destructive phase of Paget's disease with the productive phase held in abeyance. Reifenstein and Albright (1944) have supported this view, suggesting that Paget's disease always begins with an osteolytic phase. In most parts of the body the normal strains and stresses of the skeleton provoke an almost simultaneous process of repair. In the calvarium, however, no such strains exist and, therefore, the phase of repair may be indefinitely delayed

It is accepted that Paget's disease may exceptionally start in the twenties. Brailsford, for example, quotes the case of a patient aged twenty-seven. There are reports in the literature in which it is claimed that Paget's disease began below twenty, and even as early as eight years (Da Costa et al. 1915, Hummel 1934). Some of these cases are undoubtedly examples of polyostotic fibrous dysplasia. Thus the two boys of eight and eleven described by Hummel as suffering from juvenile Paget's disease had, in addition to their bone dysplasia, widespread café-au-lait pigmentation of the skin, a feature noted as characteristic by Albright ct al. (1937), who accepted these two cases as examples of polyostotic fibrous dysplasia. Other cases of Paget's disease occurring below the age of twenty include that of Elsner (1910), in which skeletal changes were observed at the age of twelve, and that of Jones (1912), whose patient first had bone changes at sixteen. It is difficult and probably profitless to discuss from this distance whether these were all genuine cases of Paget's disease or examples of fibrous dysplasia.

The precise incidence of familial Paget's disease is not easy to assess. Elmslie reviewed the ninety cases recorded up to 1908 and found seven with a family history. In 1915, when the literature included over two hundred cases, Da Costa and his colleagues estimated the familial incidence at 7 per cent. Gutman and Kasabach (1936) reported four cases of familial Paget's disease in a personal series of 104 cases. Koller (1946) was able to find only twentyseven affected families in the literature and yet he estimated the incidence of Paget's disease at 1 in 7,700 of the population of Zurich over the age of thirty. This confirms the general impression that Paget's disease is common when it is looked for, but it implies that the familial incidence is much lower than earlier work would suggest. Koller also showed that the occurrence of Paget's disease in members of the same family is due to more than chance.

Familial osteoporosis circumscripta is much rarer. I have only been able to find one example in the literature. Kasabach and Gutman (1937) described two brothers of thirty and thirty-one with widespread Paget's disease and osteoporosis circumscripta. The elder brother died at the age of thirty-one from osteogenic sarcoma of the skull.

\section{SUMMARY}

1. A father and two daughters suffering from Paget's disease are described.

2. The father's case is of special interest because he developed a malignant osteoclastoma of the mandible.

3. In the two daughters the condition was notable for the fact that in each case it began at the age of eighteen in the jaws, and led to the development of osteoporosis circumscripta of the skull.

VOL. 35 B, NO. 1, FEBRUARY 1953 
4. The relationship between Paget's disease and osteoporosis circumscripta, and the age incidence and familial incidence of Paget's disease, are discussed.

I am grateful to Dr W. N. Mann for permission to report Cases 2 and 3, and to Professor M. A. Rushton for much kindness and help in the preparation of this article, and for permission to reproduce Figures 5 and 6 . The photomicrographs were kindly provided by Mr J. E. Hutchinson. The photographs were taken by the Department of Medical Illustration, Guy's Hospital.

\section{REFERENCES}

Albright, F., Butler, A. M., Hampton, A. O., and Smith, P. (1937): Syndrome Characterized by Osteitis Fibrosa Disseminata, Areas of Pigmentation and Endocrine Dysfunction, with Precocious Puberty in Females. New England Journal of Medicine, 216, 727.

Albright, F., and Reifenstein, E. C., Jun. (1948): The Parathyroid Glands and Metabolic Bone Disease. London: Baillière, Tindall \& Cox.

Brailsford, J. F. (1938): Paget's Disease of Bone: Its Frequency, Diagnosis and Complications. British Journal of Radiology, N.S. 11, 507.

Da Costa, J. C., Funk, E. H., Bergeim, O., and Hawk, P. B. (1915): A Study of the Metabolism in Osteitis Deformans. Proceedings of the Pathological Society of Philadelphia, N.S. 17, 29.

Elmslie, R. C. (1908): Osteitis Deformans, with a Report upon Two Cases in which Sarcoma of One of the Affected Bones Arose. St Bartholomew's Hospital Reports, 44, 121.

Elsner, H. L. (1910): Osteitis Deformans (Paget's Disease) Including a Report of Two Cases. New York State Journal of Medicine, 10, 287.

Gutman, A. B., and Kasabach, H. H. (1936): Paget's Disease (Osteitis Deformans). American Journal of the Medical Sciences, 191, 361.

Hummel, R. (1934): Zwei Fälle von Ostitis deformans Paget juvenalis. Röntgen-Praxis, 6, 513.

Hunter, D., and JuPE, M. H. (1950): In A Text-Book of X-ray Diagnosis, edited by S. C. Shanks and P. Kerley. Second edition, Vol. 4. London: H. K. Lewis \& Co. Ltd.

Jones, S. F. (1912): Osteitis Deformans (Paget's Disease). Medical Record, 82, 1,155.

Kasabach, H. H., and Gutman, A. B. (1937): Osteoporosis Circumscripta of the Skull and Paget's Disease. American Journal of Roentgenology, 37, 577.

Koller, F. (1946): Úber die Heredität der Ostitis deformans Paget. Helvetica Medica Acta A, 13, 389.

PAGET, J. (1889): Remarks on Osteitis Deformans. Illustrated Medical News, 2, 181.

Reifenstein, E. C., Jun., and Albright, F. (1944): Paget's Disease: its Pathologic Physiology and the Importance of this in the Complications Arising from Fracture and Immobilization. New England Journal of Medicine, 231, 343.

Rushton, M. A. (1947): Regional Osteitis Fibrosa Affecting the Facial Bones: Two Cases. Proceedings of the Royal Society of Medicine (Section of Odontology), 40, 316.

SchmoRL, G. (1932): Úber Ostitis deformans Paget. Virchow's Archiv für Pathologische Anatomie, 283, 694. SNAPPer, I. (1949): Medical Clinics on Bone Diseases. Second edition. New York: Interscience Publishers Inc.

Sosman, M. C. (1927): Radiology as an Aid in the Diagnosis of Skull and Intracranial Lesions. Radiology, 9, 396.

Windholz, F. (1945): Osteoporosis Circumscripta Cranii: Its Pathogenesis and Occurrence in Leontiasis Ossea and in Hyperparathyroidism. Radiology, 44, 14. 